\title{
A GENERALIZATION OF THE BERNOULLI POLYNOMIAL OF ORDER ONE*
}

\author{
BY B. F. KIMBALL
}

1. Introduction. The idea of a Bernoulli polynomial, generalized so as to allow of a continuous variation of the index of its degree, is not a new one. $\dagger$ In the present paper the writer has developed a simple definition of a generalized Bernoulli polynomial of order one which brings the Bernoulli polynomial into direct relationship with the generalized Riemann zeta-function. Another very interesting property of this generalization of the Bernoulli polynomial is brought out in $\$ 7$.

2. Difference Equation Considered. For the purposes of this paper in dealing with a complex power $s$ of a complex number $t$, if $t=r e^{i \theta}$, then $t^{s} \equiv t^{\sigma+i r}=e^{s[\log r+i \theta]},(-\pi<\theta \leqq \pi)$. The following difference equation will frequently be referred to:

$$
\frac{1}{w}[f(x+w, s)-f(x, s)]=s x^{s-1},
$$

where the difference interval $w$ is taken real and positive. Also there will be occasion to impose the asymptotic condition: for any value of $s$ such that $R(s)<0$,

$$
f(x, s) \rightarrow 0 \quad \text { as } \quad R(x) \rightarrow+\infty .
$$

There will also be occasion to refer to the following regions on the $x$ and $s$ planes:

Region $X$. All points on the $x$ plane other than the negative axis of reals and the origin.

Region $S$. All points on the $s$ plane within and on the boundary of a circle of radius $M$ centering at the origin, (usually taken arbitrarily large, see \$5).

\section{Uniqueness of an Analytic Solution.}

* Presented to the Society, June 23, 1933.

† See N. E. Nörlund, Vorlesungen über Differenzenrechnung, p. 53. Future references to this book will be indicated by the letter $\mathrm{N}$. 
LEMma 1. Given a function of $x$ and $s$ which is defined for all values of $x$ on region $X$ and which for any such value of $x$ is analytic in $s$ over region $S$. Given also that this function is a solution of the difference equation (1) for $x$ in $X$ and that it satisfies the asymptotic condition (2). Such a function is unique (the existence of such a function is established in \$5).

Proof. Assuming the existence of two such functions $f_{1,2}(x, s)$, we have

$$
\psi(x+w, s)-\psi(x, s)=0, \quad\left(x \subset X ; \psi \equiv f_{1}-f_{2}\right) .
$$

From (2) it follows that $\psi(x, s)$ must be identically zero, $x \subset X$, if $R(s)<0$. Let $x_{0}$ denote any value of $x$ in region $X$. We have then from the hypothesis of Lemma 1 that $\psi\left(x_{0}, s\right)$ is an analytic function of $s$ over the region $S$. Thus this analytic function must be zero for all values of $s$ in $S$, and the lemma follows.

4. Definition of a Generalized Bernoulli Polynomial. For $s=\mathrm{a}$ positive integer or zero and $x$ real, the classical Bernoulli polynomial, $B_{s}(x) \equiv B_{s}(x, 1)$, has been defined (N., p. 18) as the polynomial solution of the difference equation (3), with difference interval equal to unity, which takes on the value of the corresponding Bernoulli number $B_{s}$ when $x=0$. Nörlund has termed the polynomial above the "Bernoulli polynomial" (of order one), with difference interval equal to one, and has defined Bernoulli polynomials of positive and negative order $n$ for all integral values of $n$, where the difference interval $w$ is not restricted to be equal to unity (N., pp. 129, 138).

The author has found it of interest to set up a definition of a generalized Bernoulli polynomial $B_{s}(x, w)$ of order one where $s$ and $x$ are allowed to take on complex values. To avoid complications, $w$ has been taken as real and positive.

Definition. The solution of the difference equation (1) described in the statement of Lemma 1 shall be considered as the generalized Bernoulli polynomial $B_{s}(x, w)$ of order one for $s$ in region $S$.

5. Solution Based on Euler-Maclaurin Summation Formula. A solution based upon the Euler-Maclaurin summation formula will first be set up. We define a periodic function $\bar{B}_{m}(t)$ as follows (N., p. 30). Let the variable $t$ be restricted to real values 
and denote by $B_{m}(t)$ the classical Bernoulli polynomial of degree $m$. The function $\bar{B}_{m}(t)$ is taken so that

$$
\bar{B}_{m}(t) \equiv B_{m}(t), \quad(0 \leqq t<1),
$$

and $\bar{B}_{m}(t+1)-\bar{B}_{m}(t)=0$ for all values of $t$. Let $m$ be any positive integer greater than a positive number $M$ (radius of circular region $S)$. Define the function $f(x, s) \equiv F(x+h w, s)$ by the relation (see N., p. 52):

$$
\begin{aligned}
& F(x+h w, s)=\sum_{k=0}^{m} w^{k} B_{k}(h) C_{s, k} x^{s-k} \\
& +(m+1) w^{m+1} C_{s, m+1} \int_{0}^{\infty} \bar{B}_{m}(h-z)(x+w z)^{s-m-1} d z, \\
& (0 \leqq h<1) .
\end{aligned}
$$

It is not difficult to verify the following statements concerning this function for $x$ in region $X$ and $s$ in region $S$.

(i) For any fixed value of $x$ in region $X, f(x, s)$ exists and is an analytic function of $s$ in $S$.

(ii) The function $f(x, s)$ defined above is a solution of the difference equation (1).

This is easily verified as follows. Consider the infinite integral as a sum of an infinite series of integrals with limits at $0, w, 2 w, \cdots$. Then take the difference of each side of the equation with respect to $x$ with difference interval $w$. This reduces the infinite series of integrals to one integral, and the expression on the right is easily recognized to be the Euler-Maclaurin expansion of $s(x+h w)^{s-1}$ in terms of its first differences.

(iii) For any value of $s$ such that $R(s)<0, f(x, s) \rightarrow 0$ as $R(x) \rightarrow+\infty$.

(iv) The function $f(x, s)$ is the generalized Bernoulli polynomial, $B_{s}(x, w)$, of order one for $s$ in region $S$.

(v) If $w=1$ and $s$ is a positive integer (or zero) less than $M$, $f(x, s)$ reduces to the classical Bernoulli polynomial.

(vi) The above region of definition $S$ of $B_{s}(x, w)$ on the $s$ plane may be made as large as desired by giving to $m$ a sufficiently large value in equation (5). Thus we have here a proof 
of the existence of $B_{s}(x, w)$ and its analyticity with respect to $s$ over the finite $s$ plane for $x \subset X$.

(vii) Set $h=0$ and $x=w$ in (5). This brings out the relation (N., p. 129)

$$
B_{s}(w, w)=w^{s} B_{s}(1,1) \equiv w^{s} B_{s}(1) .
$$

6. Multiplication Equation Satisfied by* $B_{s}(x, w)$. The difference equation

$$
h_{s}(x+w / n)-h_{s}(x)=w s x^{s-1}, \quad(x \subset X),
$$

is satisfied by $\sum_{k=0}^{n-1} B_{s}\left(x+k w / n\right.$, w) and $n^{1-s} B_{s}(n x, w)$. When $R(s)<0$ the asymptotic value of each of these functions, as $R(x)$ becomes positively infinite, is zero. Thus, with the reasoning employed in the proof of Lemma 1 , it follows that

$$
n^{1-s} B_{s}(n x, w)=\sum_{k=0}^{n-1} B_{s}(x+k w / n, w), \quad(x \subset X),
$$

for all values $\dagger$ of $s$. In particular for $n=2$ and $x=w / 2$, this gives the relation

$$
B_{s}(w / 2, w)=\left(2^{1-s}-1\right) B_{s}(w, w),
$$

which taken in connection with (6), leads to

$$
B_{s}(w / 2, w)=w^{s} B_{s}(1 / 2) .
$$

7. An Interesting Property of $B_{s}(x, w)$. Define function $\phi_{s}(x)$ by the relation:

$$
\phi_{s}(x) \equiv B_{s}(w / 2-x, w)-e^{\pi i s} B_{s}(w / 2+x, w) .
$$

Using the fact that $B_{s}(x, w)$ is a solution of the difference equation (3), one arrives at the conclusion that

$$
\phi_{s}(x+w)-\phi_{s}(x)=0, \quad(x \subset X) .
$$

When $s$ is a positive integer or zero, it follows from well known properties of the classical Bernoulli polynomials that $\phi_{s}(x)$ is identically zero for all $x$. Thus, (i) $\phi_{s}(x)$ is a periodic function

* See Thiruvenkatacharya, On some properties of the zeta function, Journal of the Indian Mathematical Society, vol. 19 (1931), pp. 92-96.

$\dagger$ In $\S 5$ the existence of $B_{s}(x, w)$ and its analyticity with respect to $s$ over the finite $s$ plane was proved. 
with period $w$ for all values of $s$; (ii) when $s$ is a positive integer or zero, $\phi_{s}(x)$ is zero for all values of $x$; (iii) $\phi_{s}(0)$ is given by

$$
\phi_{s}(0)=\left(1-e^{\pi i s}\right) B_{s}(w / 2, w)=\left(1-e^{\pi i s}\right) w^{s} B_{s}(1 / 2) .
$$

These properties point to a significant role played by $B_{s}(1 / 2)$ in the behavior* of $B_{s}(x, w)$.

8. A Closely Related Integral. Consider the integral $\dagger L(x, s)$.

$$
\begin{aligned}
& L(x, s)=\frac{w}{2 \pi i} \int_{-i \infty}^{+i \infty}(x+z)^{s}\left(\frac{\pi}{w \cos \pi z / w}\right)^{2} d z \\
&(R(x)>0) .
\end{aligned}
$$

We may write $L(x+w, s)$ in the form

$$
L(x+w, s)=\frac{w}{2 \pi i} \int_{w-i \infty}^{w+i \infty}(x+z)^{s}\left(\frac{\pi}{w \cos \pi z / w}\right)^{2} d z .
$$

The integrand of these integrals is analytic as a function of $z$ over the region bounded by the two lines $R(z)=0$ and $R(z)=w$ except for a pole at $z=w / 2$. The residue of this pole is $s(x+w / 2)^{s-1}$. Hence the integral taken around the rectangle bounded by the two lines referred to above, and lines parallel to the axis of reals at $-i N$ and $+i N$ ( $N$ real and positive) comes to $w s(x+w / 2)^{s-1}$. As $I(z) \rightarrow \pm \infty$, the absolute value of the integrand goes to zero like $t^{s} e^{-c t}, t \rightarrow \infty$, uniformly for $0 \leqq R(z) \leqq w$ ( $c$ real and positive). It follows that if we let $N \rightarrow \infty$, the contributions to the circuit integral made over the above lines at $\pm i N$ approach zero. Thus, from (11) and (12), we have

$$
L(x+w, s)-L(x, s)=w s(x+w / 2)^{s-1}, \quad(R(x)>0) .
$$

Moreover, this relation is valid for all complex values of $s$. Also it is not difficult to show that for $R(s)<0, L(x, s) \rightarrow 0$ as $R(x) \rightarrow \infty$. Upon applying the reasoning used in proving Lemma 1 , it follows that $L(x, s)$ is the unique solution of difference equation (13) for $x$ on the right half-plane which satisfies the asymptotic condition (2). Hence, for $x$ on the right half-plane, $L(x, s)$ is identical with the generalized Bernoulli polynomial $B_{s}(w / 2+x, w)$.

* See N., p. 130.

$\dagger$ See N., p. 75. 
9. Relation to the Generalized Riemann Zeta-Function. The generalized Riemann zeta-function may be introduced by the relation

$$
\zeta(x, 1-s)=\sum_{k=0}^{\infty} \frac{1}{(x+k)^{1-s}}, \quad(x \subset X, R(s)<0) .
$$

For $R(x)>0$, the definition of $\zeta(x, 1-s)$ as a function of $s$ has been extended to include the finite $s$ plane and under such definition $s \zeta(x, 1-s)$ is found to be an entire function of $s . *$

On the other hand, from the difference equation satisfied by $B_{s}(x) \equiv B_{s}(x, 1)$, it follows that

$$
\sum_{k=0}^{\infty} \frac{1}{(x+k)^{1-s}}=\frac{1}{s}\left[B_{s}(x+r+1)-B_{s}(x)\right], \quad(x \subset X) .
$$

If $R(s)<0$, it is known that $B_{s}(x+r+1) \rightarrow 0$ as $r \rightarrow \infty$. Hence,

$$
\begin{aligned}
-\frac{1}{s} B_{s}(x) & =\sum_{k=0}^{\infty} \frac{1}{(x+k)^{1-s}}, & & (x \subset X, R(s)<0), \\
B_{s}(x) & =-s \xi(x, 1-s), & & (x \subset X, R(s)<0) .
\end{aligned}
$$

When $R(x)>0$, the functions on each side of this relation, considered as functions of $s$, are defined and analytic over the finite $s$ plane. Thus the above equation holds for all values of $s$, and we can state the following theorem.

THEOREM. For $R(x)>0$ and for all values of $s$, the generalized Bernoulli polynomial of order one is related to the generalized Riemann zeta-function by equation (17).

As far as the writer is aware, this is the first time that the relation (17) has been set up for values of $s$ other than integers. The fact that it has been worth while to study the zeta-function as a function of a complex variable, may, by reason of equation (17), mean that the study of a generalized Bernoulli polynomial will yield further interesting and worth while results. In passing it is interesting to note that the relation (17) may serve to extend the domain of definition of $\zeta(x, s)$, for all values of $s$, to values of $x$ over all of region $X$.

* See, for example, Whittaker and Watson, Modern Analysis, 3rd ed., Chap. 13. 
From the relation between the zeta-function and the generalized Bernoulli polynomial there emerge several interesting consequences. From the well known functional relation for $\zeta(1, s)$ it follows that

$$
s B_{1-s}(1)=2^{s} \pi^{s-1} \sin (s \pi / 2) \Gamma(2-s) B_{s}(1)
$$

for all values of $s$. Since $B_{s}(1 / 2)$ plays a central role in the general behavior of the function $B_{s}(x, w)$, it is of interest to apply the above relation to $B_{s}(1 / 2)$. From the multiplication equation (6) when we set $w=1, n=2, x=1 / 2$, we obtain

$$
B_{s}(1 / 2)=\left(2^{1-s}-1\right) B_{s}(1),
$$

which, when substituted in (18), gives

$$
B_{1-s}(1 / 2)=\frac{2^{s} \pi^{s-1}}{s}\left[\frac{2^{s}-1}{2^{1-s}-1}\right] \Gamma(2-s) \sin (s \pi / 2) B_{s}(1 / 2) .
$$

This relation holds for all values of $s$.

If $w$ were given other values than unity, a further extension of the zeta-function would result from the relation (17). This would mean that in the series definition of the zeta-function (14) the denominators $x+k$ would be replaced by numbers $x+k w$. Such a generalized zeta-function would be the solution of the difference equation

$$
\frac{1}{w}[f(x+w, s)-f(x, s)]=-x^{-s}, \quad(x \subset X),
$$

analytic in $s$ over the finite $s$ plane (except for a pole at $s=1$ ) which for $R(s)>1$ has its asymptotic value equal to zero for $R(x)$ positively infinite.

Albany, N. Y. 